Review

\title{
The Hedgehog Signaling Pathway in the Ischemic Heart, Brain, and Skeletal Muscle
}

\author{
Igor Giarretta 1, Eleonora Gaetani ${ }^{1}$, Paolo Tondi ${ }^{1}$, Takayuki Asahara ${ }^{2}$ and Roberto Pola 1,* \\ 1 Department of Medicine, Fondazione Policlinico Universitario A. Gemelli IRCCS, Università Cattolica del \\ Sacro Cuore, Rome, Italy; igor.giarretta@unicatt.it (I.G.); eleonora.gaetani@unicatt.it (E.G.); \\ paolotondi@yahoo.it (P.T.) \\ 2 Department of Regenerative Medicine Science, Tokai University School of Medicine, 143 Shimokasuya, \\ Isehara, Kanagawa 259-1193, Japan; asa777@is.icc.u-tokai.ac.jp (T.A.) \\ * Correspondence: roberto.pola@unicatt.it; Tel: +39-06-3015-7075
}

\begin{abstract}
Hedgehog (Hh) proteins are prototypical morphogens known to regulate epithelial/mesenchymal interactions during embryonic development. In addition to its pivotal role in embryogenesis, the Hh signaling pathway may be recapitulated in post-natal life in a number of physiological and pathological conditions, including ischemia. This review highlights the involvement of Hh signaling in ischemic tissue regeneration and angiogenesis, with particular attention to the heart, the brain, and the skeletal muscle. Updated information on the potential role of the Hh pathway as a therapeutic target in ischemic condition is also presented.
\end{abstract}

Keywords: Hedgehog; Ischemia; Heart; Brain; Skeletal muscle.

\section{Introduction}

Hedgehog $(\mathrm{Hh})$ signaling was first described in 1980 by Nusslein-Volhard and Wieschaus and was initially addressed as a key mediator of cellular proliferation, differentiation, and migration during organogenesis in invertebrates [1]. The general signaling mechanism for the Hh pathway is conserved from fly to mammal although more and distinct components have been found in mammalian cells [2]. In the early 1990s, three Hh homologs were discovered in vertebrates: Sonic (Shh), Desert (Dhh), and Indian hedgehog (Ihh). Among them, Shh is the most widely expressed during development and the best studied. The initial evidence for the importance of Hh signaling in mammalian development was provided by observations that mutations in Shh cause holoprosencephaly, a developmental disorder that affects midline morphogenesis of the face and nervous system [3-5]. Although most studies of Hh signaling focus on Shh, the other two ligands Dhh and Ihh - share many of the same downstream signaling components. The Dhh ligand is more abundant in the reproductive organs of both males and females, including Sertoli cells of testes and granulosa cells of ovaries [6, 7]. Consistent with this, male mice lacking Dhh are infertile due to the complete absence of mature sperm [6]. Ihh plays instead an important role in skeletal development, primarily in cortical bone and long bone formation. The most severe manifestation of Ihh deficiency may be seen in children with acrocapitofemoral dysplasia, who have short stature and bone defects [8].

Canonically, $\mathrm{Hh}$ signaling occurs through binding of $\mathrm{Hh}$ proteins to the twelvetransmembrane domain receptor Patched (Ptch). Binding of a Hh ligand to Ptch results in Ptch internalization and abolishment of its inhibitory activity on the seven-transmembrane GPCR-like protein Smoothened (Smo). Smo is subsequently phosphorylated at its intracellular C-terminus and undergoes reciprocal translocation to the plasma membrane of non-motile cilia, inhibiting the downstream proteolytic processing of GLI2 and GLI3, leading to the production of full-length activated Gli proteins (GliA). GliA enter the nucleus and stimulate transcription of the ubiquitous Hh target genes, GLI1, PTCH, and HHIP (Hh interacting protein). Signal transmission is then controlled through a complex signaling cascade that results in a balance between activator (GliA) 
and repressor (GliR) proteins [9]. In addition to this canonical pathway, there are cellular and tissue responses to Hh ligands that are independent of transcriptional changes mediated by the Gli family. These pathways may be important for apoptosis, cell duplication, differentiation, and determination. For instance, it has been reported that Smo modulates actin cytoskeleton-dependent processes, such as fibroblast migration, endothelial cell tubulogenesis, axonal extension, and neurite formation, by mechanisms that exclude Gli-dependent transcription [10].

It was 2001 when we first reported that the Hedgehog $(\mathrm{Hh})$ signaling pathway has a role in angiogenesis [11]. Before that seminal publication, there were only sparse observations pointing to the possible involvement of $\mathrm{Hh}$ in vascularizing certain embryonic tissues. For instance, it was known that hypervascularization of the neuroectoderm occurs upon transgenic overexpression of Shh in the dorsal neural tube [12], or that Shh-deficient zebrafish has disorganization of endothelial precursors and inability to form the dorsal aorta or axial vein [13], or that Shh-deficient mice lack proper vascularization of the developing lung [14]. Nowadays, there is substantial evidence that the Hh signaling pathway is an important player in the regulation of angiogenesis in a number of physiological and pathological conditions in post-natal life, with multiple implications in the field of cardiovascular medicine, neurology, and oncology. This review presents an updated summary of our understanding of the Hh signaling pathway in the regulation of angiogenesis, with a specific focus on Hh-mediated effects at the level of the ischemic heart, brain, and skeletal muscle.

\section{Hedgehog in the ischemic skeletal muscle}

In the adult vasculature, adventitial cells, endothelial cells, and cells in the medial layer normally express the Hh receptor Ptch, indicating that adult cardiovascular tissues are ready to respond to $\mathrm{Hh}$ ligands in post-natal life [11]. One condition that leads to such event, with overexpression of Shh and upregulation of Ptch, is ischemia of the skeletal muscle [15]. In this setting, Shh and Ptch upregulation take place in the same cell type, i.e. interstitial mesenchymal cells within the ischemic area, indicating the presence of an autocrine mechanism. These cells produce vascular endothelial growth factor (VEGF) under ischemic conditions, through a Shhdependent mechanism, as demonstrated by the evidence that VEGF upregulation is reduced in mice treated with a Shh-blocking antibody after induction of ischemia [15].

The fact that Shh modulates VEGF production in ischemic tissues is one of the fundamental mechanisms through which the Hh pathway regulates angiogenesis. Indeed, we have originally reported that Shh has no direct effect on endothelial cell migration or proliferation [11]. Instead, Shh induces expression of angiogenic cytokines, including all three VEGF-1 isoforms and angiopoietins- 1 and -2, from interstitial mesenchymal cells, thus acting as an indirect angiogenic agent [11]. The ability of Shh to induce the production of angiogenic growth factors, including VEGF, HGF, PDGF-BB, and IGF, in interstitial cells and fibroblasts has been later confirmed by other authors [16-19]. Also, it has been confirmed that Hh ligands are unable to induce Gli-target genes in endothelial cells and that Shh has limited effects on endothelial cell proliferation and migration [20,21]. There is also the demonstration that Gli3 deficiency in endothelial cells does not affect repair of the ischemic skeletal muscle [22]. On the other hand, it has been reported that $\mathrm{Hh}$ proteins have the ability to promote endothelial cell tubulogenesis through a non-canonical $\mathrm{Hh}$ pathway that involves Smo, Gi proteins, and Rac1 and results in cytoskeletal rearrangement and formation of actin stress fibers in endothelial cells [20]. Nonetheless, endothelial-specific Smo knockout (eSmoNull) mice have no observable phenotype and display unaltered ability to mount an appropriate angiogenic process in response to hindlimb ischemia [23]. Even Shh-induced corneal angiogenesis is normal in eSmoNull mice [23]. Taken together, these findings highlight the complexity of the Hh pathway and indicate that further studies are needed to fully understand the molecular mechanisms responsible for Hh-regulated angiogenesis.

Some controversies also exist on the identity of the Hh ligand that plays the most important role during ischemia-induced angiogenesis. We have found that hindlimb ischemia results in upregulation of Shh expression, without detectable effect on Ihh and Dhh [15]. However, more recent findings indicate that limb perfusion is significantly impaired in the absence of Dhh, due to 
the fact that Dhh is important for peripheral nerve survival in the ischemic muscle and the maintenance of the pool of nerve-derived proangiogenic factors [24]. In addition, the rescue of Dhh expression by gene therapy in old mice promotes ischemia-induced angiogenesis [24].

If, on one hand, it is still not completely clear how Hh regulates angiogenesis, on the other hand it is generally accepted that stimulation of the Hh pathway may help revascularization and repair of ischemic tissues. Indeed, Shh protein, injected intraperitoneally in aged mice, augments blood-flow recovery and limb salvage following operatively induced hind-limb ischemia [11]. Also, intramuscular treatment with a plasmid encoding the Shh human gene (phShh) increases blood flow, capillary density, and arteriole density in old mice in which peripheral circulation of the hindlimb has been disrupted by removal of the common femoral artery, or in which the skeletal muscle has been mechanically injured $[25,26]$. Shh gene therapy also enhances vasculogenesis, by increasing the number of circulating bone marrow (BM)-derived endothelial progenitor cells (EPCs) and improving the contribution of these cells to the process of neovascularization [27]. Combined therapy with Shh gene transfer and BM-derived EPCs is more effective than Shh gene therapy alone in an experimental model of peripheral limb ischemia [28]. The importance of the Hh pathway in the pathophysiology of the EPC compartment has been recently further strengthened by the demonstration that Gli1 and Ptch expression are reduced in the EPCs of streptozotocin (STZ)induced type 1 diabetic mice and that administration of a Shh pathway receptor agonist restores both the number and function of EPCs and increases neovascularization in these mice in response to ischemia [29]. Finally, as mentioned above, there are data supporting a potential therapeutic role also for Dhh in the ischemic skeletal muscle [24].

A summary of the results of the studies conducted on the ischemic skeletal muscle is presented in Table 1. 
Table 1. Hedgehog signaling in the ischemic skeletal muscle.

Result $\quad$ First author $\begin{aligned} & \text { Journal and year of } \\ & \text { publication }\end{aligned}$ Reference

$\begin{array}{lll}\text { Hindlimb ischemia induces Shh and Ptch upregulation } \quad \text { Pola } & \text { Circulation, } 2003 \quad 15\end{array}$
in mice

Hh inhibition leads to decreased upregulation of VEGF Pola Circulation, 2003 in response to ischemia

15

Ablation of the Hh pathway in endothelial cells does not Gupta $\quad$ Lab Invest, 2018

affect angiogenic response to ischemia in mice

Gli3-deficient mice have reduced capillary density after Renault $\quad$ Circulation Res, 2009 induction of hindlimb ischemia

Dhh KO mice have impaired angiogenesis in response to Renault Circulation Res, 2013 ischemia

Intraperitoneal injection of Shh protein enhances

Pola

Nature Med, 2001

11

angiogenic response to ischemia in aged mice

Intramuscular treatment with a plasmid encoding the

Straface

J Cell Mol Med, 2009

25

Shh human gene (phShh) enhances angiogenic response

to ischemia in aged mice

Intramuscular treatment with phShh enhances skeletal Piccioni muscle regeneration in aged mice

J Gerontol A Biol Sci

26

Med Sci, 2014

Intramuscular treatment with phShh increases the

Palladino

Molecular Therapy, 2011

15

number of bone marrow (BM)-derived endothelial progenitor cells (EPCs) in response to ischemia in mice

Combined therapy with phShh and BM-derived EPCs enhances angiogenesis and myogenesis in the murine Palladino

ischemic skeletal muscle

Gli1 and Ptch expression are reduced in the EPCs of diabetic mice

Qin

Mol Cell Endocrinol, 2016

Treatment with a Shh receptor agonist restores number

Qin

Mol Cell Endocrinol, 29 and function of EPCs in diabetic mice and increases 2016 neovascularization in response to ischemia 


\section{Hedgehog in the ischemic heart}

Hh signaling seems to have a crucial homeostatic role in the adult heart. This has been elegantly demonstrated by Lavine and coll., who have ablated endogenous Hh signaling in murine myocardial and perivascular smooth muscle cells, finding that, in the absence of Hh, there is loss of coronary blood vessels, tissue hypoxia, cardiomyocyte cell death, heart failure, and increased lethality [30]. However, a study by Paulis and coll. has shown that, although Ptch and Smo are expressed in adult cardiomyocytes, inhibition of the endogenous Hh does not result in significant electrocardiographic (ECG) changes, suggesting that active Hh signaling is not important in the adult heart [31]. This apparent contradiction between the two studies might depend on the fact that, in the latter study, inhibition of the Hh pathway was achieved by infusing the Hh antagonist cyclopamine for only 6 hours before ECG analysis, while in the study of Lavine and coll. the inhibition of the pathway lasted several days and was obtained by ablating Smo in specific cell types.

During myocardial ischemia, there is a strong upregulation of Shh and Ptch in the adult mouse heart [32-34]. Xiao and coll. have reported that, in streptozotocin-induced type 1 diabetic mice, myocardial expression of Shh, Ptch, and Gli1 is significantly decreased, and this is accompanied by cardiac dysfunction [32]. In these mice, treatment with a Shh pathway agonist significantly increases Ptch and Gli1 proteins, enhances capillary density, reduces the percentage of myocardial infarction size, and ameliorates cardiac function. These findings indicate that in type 1 diabetic mice the myocardial Shh pathway is impaired and that strategies aimed at stimulating the Shh pathway may be beneficial for improving cardiac dysfunction.

Taken together, these results suggest that $\mathrm{Hh}$ signaling has a protective role in response to cardiac ischemia. Based on this, several experimental studies have tested the possibility to enhance angiogenesis and improve heart function after myocardial ischemia, by acting on the Hh pathway. Our group has used intramyocardial gene transfer of naked DNA encoding human Shh (phShh) to enhance neovascularization, reduce fibrosis, and preserve left ventricular function in rabbit and pig models of acute and chronic myocardial ischemia [33]. Ahmed and coll. have overexpressed Shh in rat mesenchymal stem cells (MSCs) [35]. The consequence has been increased expression of angiogenic and pro-survival growth factors in transfected cells, along with improved ability to migrate and form tubes in vitro. When injected in the ischemic myocardium, Shh-overexpressing MSCs lead to increased density of functionally competent blood vessels and preserve function of the left ventricle. Guo and coll. have overexpressed Shh in cardiac microvascular endothelial cells (CMECs) isolated from rat heart tissues [36]. This has resulted in increased CMEC viability and decreased apoptosis, along with increased production of angiogenic factors, including VEGF, FGF and angiopoietins. In a rat model of ischemia-reperfusion, stimulation of Shh pathway prior to reperfusion has reduced both infarct size and subsequent arrhythmias by preventing ventricular repolarization abnormalities [31]. This is an interesting cardio-protective effect of Shh acting directly on cardiomyocytes.

The angiogenic effects of Shh in the ischemic heart appear to be mediated by the canonical Glidependent pathway. Indeed, surgically induced myocardial infarction results in reduced capillary density and left ventricular ejection fraction in Gli3-haploinsufficient (Gli3(+/-)) mice, compared to wild-type mice [21]. Gli3-deficient mice also display reduced capillary density after induction of hindlimb ischemia and impaired angiogenic response to VEGF in the corneal angiogenesis model [21]. Also, a microarray analysis of gene expression changes elicited by Shh and sensitive to a Smo inhibitor has led to the identification of a subset of 37 cardiomyocyte-specific genes regulated by 
Shh, including some in the PKA and purinergic signaling pathways [37]. In addition, neonatal rat ventricular cardiomyocytes infected with an adenovirus encoding GiCT, a peptide that impairs receptor-Gi protein coupling, showed reduced activation of Hh targets. In vitro data were confirmed in transgenic mice with cardiomyocyte-inducible GiCT expression [37]. Transgenic GiCT mice showed specific reduction of Gli1 expression in the heart under basal conditions and failed to upregulate the Hh pathway upon ischemia and reperfusion injury [37]. This study characterizes the transcriptional response of cardiomyocytes to Shh and establishes a critical role for Smo coupling to $\mathrm{Gi}$ in Hh signaling in the normal and ischemic myocardium.

The results of the studies conducted on the ischemic heart are summarized in Table 2.

Table 2. Hedgehog signaling in the ischemic heart.

\begin{tabular}{|c|c|c|c|}
\hline Result & First author & $\begin{array}{l}\text { Journal and year of } \\
\text { publication }\end{array}$ & Ref. \\
\hline \multirow{3}{*}{$\begin{array}{l}\text { Ischemia induces upregulation of Shh and Ptch in } \\
\text { the adult mouse heart }\end{array}$} & Xiao & Cardiovasc Res, 2012 & 32 \\
\hline & Kusano & Nature Med, 2005 & 33 \\
\hline & Bijlsma & Exp Biol Med, 2008 & 34 \\
\hline
\end{tabular}

Gli3-deficient mice have reduced capillary density Renault $\quad$ Circulation Res, 2009

21 and left ventricular ejection fraction after myocardial infarction

Upregulation of Shh, Ptch, and Gli1 is impaired in $\quad$ Xiao
the ischemic myocardium of diabetic mice

Treatment with Shh pathway agonist enhances
capillary density, reduces infarct size, and improves

Xiao Cardiovasc Res, 2012

32

cardiac function in diabetic mice with myocardial

infarction

Intramyocardial gene transfer of phShh enhances

Kusano

Nature Med, 2005

33

neovascularization, reduces fibrosis, and preserves

left ventricular function in rabbit and pig models of

acute and chronic myocardial ischemia

Injection in the rat ischemic myocardium of Shh-

Ahmed

PLoS One, 2010

35

overexpressing mesenchymal stem cells (MSCs)

increases density of functionally competent blood

vessels and preserves function of the left ventricle

In a rat model of ischemia-reperfusion, stimulation Paulis

Sci Rep, 2015

31

of Shh pathway prior to reperfusion reduces infarct

size and arrhythmias

\section{Hedgehog in the ischemic brain}

A number of data support the concept that $\mathrm{Hh}$ signaling is an important regulator of neurogenesis and angiogenesis in the adult brain. For instance, it is known that Shh is required for 
the maintenance of neural progenitor cells (NPCs) in the adult hippocampus and regulates the proliferation of NPCs after ischemia/hypoxia [38]. Middle cerebral artery occlusion is associated with increased mRNA encoding both Shh and its transcription factor Gli1, already a few hours after induction of brain ischemia [38]. By employing a co-culture system of brain microvascular endothelial cells (BMECs) and astrocytes, incubated under oxygen-glucose deprivation (OGD) condition, it has been demonstrated that Shh is secreted by activated astrocytes and promotes proliferation, migration, and tube formation of BMECs [39]. In this assay, proliferation, migration, and tube formation of BMECs may be suppressed by silencing the Ras homolog gene family member A (RhoA) and blocking Rho-dependent kinase (ROCK) [39]. Not only ODG, but also oxidative stress has been shown to have the ability of activating the Shh pathway in cultured cortical neurons [40]. In this setting, activation of endogenous Shh signaling protects neurons from $\mathrm{H}_{2} \mathrm{O}_{2}$-induced apoptosis and increases cell viability. In addition, in $\mathrm{H}_{2} \mathrm{O}_{2}$-treated neurons, exogenous Shh increases the activities of Superoxide dismutase (SOD) and Glutathione peroxidase (GSH-PX) and decreases production of Malondialdehyde (MDA). It also promotes expression of the anti-apoptotic gene Bcl-2 and inhibits expression of pro-apoptotic gene Bax. Activation of Shh signal also leads to upregulated expression of both neurotrophic and vascular factors, such as VEGF and brain-derived neurotrophic factor (BDNF) [40, 41].

The importance of endogenous Shh upregulation in the ischemic brain is demonstrated by the fact that, upon induction of experimental cerebral ischemia, inhibition of the Shh pathway results in increased infarct volume, brain water content, and behavioral deficits [42]. The endogenous $\mathrm{Hh}$ pathway appears to be important also for the beneficial effects induced by bone marrow stromal cells (MSCs) on neurologic recovery after middle cerebral artery occlusion in mice [43]. Indeed, treatment with MSCs significantly enhances functional recovery after middle cerebral artery occlusion, concurrent with increases of synaptophysin, synapse density, and myelinated axons along the ischemic boundary zone. However, these benefits are significantly reduced by blockage of the endogenous Shh pathway [43]. Also, the beneficial effects displayed by cerebrolysin - a mixture of neurotrophic peptides - in experimental neurodegenerative diseases and stroke seem to depend on proper activity of the endogenous Hh pathway [44]. Indeed, cerebrolysin significantly increases neural progenitor cell proliferation and differentiation into neurons and myelinating oligodendrocytes in rats subjected to embolic stroke. However, blockage of the Shh signaling pathway with a pharmacological smoothened inhibitor, cyclopamine, abolishes cerebrolysininduced in vitro neurogenesis and oligodendrogenesis. Moreover, profound neurological function improvements were observed in rats treated with cerebrolysin from week 3 to week 5 after stroke onset. However, in vivo inhibition of the Shh pathway with cyclopamine completely reverses the effects of cerebrolysin on functional recovery.

Taken together, these finding suggest a potential role for Shh in the treatments of brain ischemia. Based on that, Chen and coll. have performed intracerebroventricular injections of Shh in rats immediately after permanent middle cerebral artery occlusion [17]. This treatment has reduced infarct volume, led to improved microvascular density and neuron survival in the ischemic boundary zone, and improved neurological scores. These beneficial effects occurred along with enhanced VEGF expression [17]. Stimulation of the Shh pathway appears to be beneficial even if performed not immediately after stroke. Indeed, delayed treatment (1 month after stroke) with a Shh agonist in mice resulted in enhanced functional recovery both in locomotor and cognitive function [45]. Furthermore, using a genetically inducible neural stem cell-specific reporter mouse line (nestin-CreERT2-R26R-YFP), which allows to label and track neural stem cell proliferation, survival, and differentiation in ischemic brain, it was shown that post-stroke treatment with a Shh pathway agonist increases survival of newly born cells derived from both subventricular and subgranular zone and neurons in the ischemic brain [45]. Huang and coll. have tested whether epidural application of the biologically active N-terminal fragment of Shh (Shh-N) may reduce the extent of ischemic brain injury in rats exposed to a 60-min episode of middle cerebral artery occlusion. Shh-N was applied topically, by placing a fibrin glue over the peri-infarct cortex [46]. They found that such treatment substantially reduced infarct volumes after 7 days of reperfusion. It 
also improved behavioral outcomes as assessed by global neurological functions, rotarod test, and grasping power test. Furthermore, Shh-N attenuated the extents of protein oxidation, lipid peroxidation, and apoptosis induced by focal ischemia/reperfusion. Immunohistochemical staining revealed that Shh-N enhanced post-ischemic angiogenesis, stimulated the proliferation of nestinpositive neural progenitor cells (NPCs), and suppressed astrocytosis.

The results of the studies conducted on the ischemic brain are summarized in Table 3. 
Table 3. Hedgehog signaling in the ischemic brain.

\begin{tabular}{|c|c|c|c|}
\hline Result & First author & $\begin{array}{l}\text { Journal and year of } \\
\text { publication }\end{array}$ & Ref. \\
\hline $\begin{array}{l}\text { Middle cerebral artery occlusion induces } \\
\text { upregulation of Shh and Gli1 in the mouse brain }\end{array}$ & Sims & Stroke, 2009 & 38 \\
\hline $\begin{array}{l}\text { Inhibition of the Hh pathway suppresses ischemia- } \\
\text { induced proliferation of subgranular neural } \\
\text { progenitor cells (NPCs) in mice }\end{array}$ & Sims & Stroke, 2009 & 38 \\
\hline $\begin{array}{l}\text { Inhibition of the Hh pathway after permanent } \\
\text { middle cerebral artery occlusion increases infarct } \\
\text { volume, brain water content, and behavioral } \\
\text { deficits in rats }\end{array}$ & $\mathrm{Ji}$ & Neurosci Lett, 2012 & 42 \\
\hline $\begin{array}{l}\text { Inhibition of the Hh pathway reduces the } \\
\text { beneficial effects of treatment with bone marrow } \\
\text { stromal cells (MSCs) after middle cerebral artery } \\
\text { occlusion in mice }\end{array}$ & Ding & $\begin{array}{l}\text { J Cereb Blood Flow } \\
\text { Metab, } 2013\end{array}$ & 43 \\
\hline $\begin{array}{l}\text { Inhibition of the Hh pathway suppresses the } \\
\text { beneficial effects of cerebrolysin - a mixture of } \\
\text { neurotrophic peptides - in experimental models of } \\
\text { neurodegenerative diseases and stroke }\end{array}$ & Zhang & Stroke, 2013 & 44 \\
\hline $\begin{array}{l}\text { Intracerebroventricular injections of Shh in rats } \\
\text { immediately after permanent middle cerebral } \\
\text { artery occlusion reduces infarct volume, enhances } \\
\text { microvascular density and neuron survival in the } \\
\text { ischemic boundary zone, and improves } \\
\text { neurological scores }\end{array}$ & Chen & Neuroscience, 2017 & 17 \\
\hline $\begin{array}{l}\text { Delayed treatment ( } 1 \text { month after stroke) with a } \\
\text { Shh agonist enhances functional recovery in } \\
\text { locomotor and cognitive function in mice }\end{array}$ & Jin & Stroke, 2017 & 45 \\
\hline $\begin{array}{l}\text { Post-stroke treatment with a Shh agonist increases } \\
\text { surviving newly born cells derived from the } \\
\text { subventricular and subgranular zone and neurons } \\
\text { in the mouse ischemic brain }\end{array}$ & Jin & Stroke, 2017 & 45 \\
\hline
\end{tabular}


7 of 14

Epidural application of the biologically active N-

Huang

Exp Neurol, 2013

46 terminal fragment of Shh (Shh-N) enhances angiogenesis, stimulates proliferation of nestinpositive neural progenitor cells (NPCs), suppresses astrocytosis, reduces infarct volume, and improves behavioral outcomes in rats exposed to a $60-\mathrm{min}$ episode of middle cerebral artery occlusion 


\section{Concluding Remarks}

Once believed to be important only during embryonic development, the Hh signaling pathway is now recognized as a crucial player in many physiological and pathological conditions in postnatal life. Its role during ischemia-induced angiogenesis and tissue repair has been extensively investigated in the last two decades and seems to be particularly important in organs such as the heart, the brain, and the skeletal muscle. Although the precise molecular and cellular mechanisms underlying Hh-mediated angiogenesis still require to be fully elucidated, there is substantial evidence supporting the concept that the endogenous Hh signaling pathway has the ability to orchestrate multiple aspects of the angiogenic response to ischemia, mainly through the production of angiogenic growth factors from interstitial cells and fibroblasts and the regulation of EPC activity. Nevertheless, there is evidence that many activities of Hh proteins in the ischemic heart and brain occur through direct stimulation of cardiomyocytes and neural cells. Based on the concept that the endogenous $\mathrm{Hh}$ pathway is important for efficient angiogenic response to ischemia, several studies have focused on the use of agonists of the Hh pathway to stimulate therapeutic angiogenesis. Successful results have been achieved in experimental models of myocardial infarction, ischemic stroke, and peripheral limb ischemia, but further studies are needed to fully understand the translational significance and potential clinical implications of these findings.

Author Contributions: I.G., E.G., P.T., T.A. and R.P. reviewed the literature and wrote the manuscript.

Funding: This research received no external funding

Conflicts of Interest: The authors declare no conflict of interest.

\section{References}

1. Nusslein-Volhard, C., and E. Wieschaus. "Mutations Affecting Segment Number and Polarity in Drosophila." Nature 287, no. 5785 (1980): 795-801.

2. Ingham, P. W., and M. Placzek. "Orchestrating Ontogenesis: Variations on a Theme by Sonic Hedgehog." Nat Rev Genet 7, no. 11 (2006): 841-50.

3. Chiang, C., Y. Litingtung, E. Lee, K. E. Young, J. L. Corden, H. Westphal, and P. A. Beachy. "Cyclopia and Defective Axial Patterning in Mice Lacking Sonic Hedgehog Gene Function." Nature 383, no. 6599 (1996): 407-13.

4. Belloni, E., M. Muenke, E. Roessler, G. Traverso, J. Siegel-Bartelt, A. Frumkin, H. F. Mitchell, H. Donis-Keller, C. Helms, A. V. Hing, H. H. Heng, B. Koop, D. Martindale, J. M. Rommens, L. C. Tsui, and S. W. Scherer. "Identification of Sonic Hedgehog as a Candidate Gene Responsible for Holoprosencephaly." Nat Genet 14, no. 3 (1996): 353-6.

5. Roessler, E., E. Belloni, K. Gaudenz, P. Jay, P. Berta, S. W. Scherer, L. C. Tsui, and M. Muenke. "Mutations in the Human Sonic Hedgehog Gene Cause Holoprosencephaly." Nat Genet 14, no. 3 (1996): 357-60.

6. Bitgood, M. J., L. Shen, and A. P. McMahon. "Sertoli Cell Signaling by Desert Hedgehog Regulates the Male Germline." Curr Biol 6, no. 3 (1996): 298-304.

7. Varjosalo, M., and J. Taipale. "Hedgehog: Functions and Mechanisms." Genes Dev 22, no. 18 (2008): 2454-72.

8. Hellemans, J., P. J. Coucke, A. Giedion, A. De Paepe, P. Kramer, F. Beemer, and G. R. Mortier. "Homozygous Mutations in Ihh Cause Acrocapitofemoral Dysplasia, an Autosomal Recessive Disorder with Cone-Shaped Epiphyses in Hands and Hips." Am J Hum Genet 72, no. 4 (2003): 10406.

9. Carballo, G. B., J. R. Honorato, G. P. F. de Lopes, and Tclse Spohr. "A Highlight on Sonic Hedgehog Pathway." Cell Commun Signal 16, no. 1 (2018): 11.

10. Shevde, L. A., and R. S. Samant. "Nonclassical Hedgehog-Gli Signaling and Its Clinical Implications." Int J Cancer 135, no. 1 (2014): 1-6. 
11. Pola, R., L. E. Ling, M. Silver, M. J. Corbley, M. Kearney, R. Blake Pepinsky, R. Shapiro, F. R. Taylor, D. P. Baker, T. Asahara, and J. M. Isner. "The Morphogen Sonic Hedgehog Is an Indirect Angiogenic Agent Upregulating Two Families of Angiogenic Growth Factors." Nat Med 7, no. 6 (2001): 706-11.

12. Rowitch, D. H., S. Jacques B, S. M. Lee, J. D. Flax, E. Y. Snyder, and A. P. McMahon. "Sonic Hedgehog Regulates Proliferation and Inhibits Differentiation of Cns Precursor Cells." J Neurosci 19, no. 20 (1999): 8954-65.

13. Brown, L. A., A. R. Rodaway, T. F. Schilling, T. Jowett, P. W. Ingham, R. K. Patient, and A. D. Sharrocks. "Insights into Early Vasculogenesis Revealed by Expression of the Ets-Domain Transcription Factor Fli-1 in Wild-Type and Mutant Zebrafish Embryos." Mech Dev 90, no. 2 (2000): 237-52.

14. Pepicelli, C. V., P. M. Lewis, and A. P. McMahon. "Sonic Hedgehog Regulates Branching Morphogenesis in the Mammalian Lung." Curr Biol 8, no. 19 (1998): 1083-6.

15. Pola, R., L. E. Ling, T. R. Aprahamian, E. Barban, M. Bosch-Marce, C. Curry, M. Corbley, M. Kearney, J. M. Isner, and D. W. Losordo. "Postnatal Recapitulation of Embryonic Hedgehog Pathway in Response to Skeletal Muscle Ischemia." Circulation 108, no. 4 (2003): 479-85.

16. Li, Y., Y. Xia, Y. Wang, L. Mao, Y. Gao, Q. He, M. Huang, S. Chen, and B. Hu. "Sonic Hedgehog (Shh) Regulates the Expression of Angiogenic Growth Factors in Oxygen-Glucose-Deprived Astrocytes by Mediating the Nuclear Receptor Nr2f2." Mol Neurobiol 47, no. 3 (2013): 967-75.

17. Chen, S. C., M. Huang, Q. W. He, Y. Zhang, E. N. Opoku, H. Yang, H. J. Jin, Y. P. Xia, and B. Hu. "Administration of Sonic Hedgehog Protein Induces Angiogenesis and Has Therapeutic Effects after Stroke in Rats." Neuroscience 352 (2017): 285-95.

18. Chen, W., T. Tang, J. Eastham-Anderson, D. Dunlap, B. Alicke, M. Nannini, S. Gould, R. Yauch, Z. Modrusan, K. J. DuPree, W. C. Darbonne, G. Plowman, F. J. de Sauvage, and C. A. Callahan. "Canonical Hedgehog Signaling Augments Tumor Angiogenesis by Induction of Vegf-a in Stromal Perivascular Cells." Proc Natl Acad Sci U S A 108, no. 23 (2011): 9589-94.

19. Soleti, R., T. Benameur, C. Porro, M. A. Panaro, R. Andriantsitohaina, and M. C. Martinez. "Microparticles Harboring Sonic Hedgehog Promote Angiogenesis through the Upregulation of Adhesion Proteins and Proangiogenic Factors." Carcinogenesis 30, no. 4 (2009): 580-8.

20. Chinchilla, P., L. Xiao, M. G. Kazanietz, and N. A. Riobo. "Hedgehog Proteins Activate ProAngiogenic Responses in Endothelial Cells through Non-Canonical Signaling Pathways." Cell Cycle 9, no. 3 (2010): 570-79.

21. Renault, M. A., J. Roncalli, J. Tongers, S. Misener, T. Thorne, K. Jujo, A. Ito, T. Clarke, C. Fung, M. Millay, C. Kamide, A. Scarpelli, E. Klyachko, and D. W. Losordo. "The Hedgehog Transcription Factor Gli3 Modulates Angiogenesis." Circ Res 105, no. 8 (2009): 818-26.

22. Renault, M. A., J. Roncalli, J. Tongers, T. Thorne, E. Klyachko, S. Misener, O. V. Volpert, S. Mehta, A. Burg, C. Luedemann, G. Qin, R. Kishore, and D. W. Losordo. "Sonic Hedgehog Induces Angiogenesis Via Rho Kinase-Dependent Signaling in Endothelial Cells." J Mol Cell Cardiol 49, no. 3 (2010): 490-8.

23. Gupta, R., A. R. Mackie, S. Misener, L. Liu, D. W. Losordo, and R. Kishore. "Endothelial Smoothened-Dependent Hedgehog Signaling Is Not Required for Sonic Hedgehog Induced Angiogenesis or Ischemic Tissue Repair." Lab Invest 98, no. 5 (2018): 682-91.

24. Renault, M. A., C. Chapouly, Q. Yao, F. Larrieu-Lahargue, S. Vandierdonck, A. Reynaud, M. Petit, B. Jaspard-Vinassa, I. Belloc, E. Traiffort, M. Ruat, C. Duplaa, T. Couffinhal, C. Desgranges, and A. P. Gadeau. "Desert Hedgehog Promotes Ischemia-Induced Angiogenesis by Ensuring Peripheral Nerve Survival." Circ Res 112, no. 5 (2013): 762-70.

25. Straface, G., T. Aprahamian, A. Flex, E. Gaetani, F. Biscetti, R. C. Smith, G. Pecorini, E. Pola, F. Angelini, E. Stigliano, J. J. Castellot, Jr., D. W. Losordo, and R. Pola. "Sonic Hedgehog Regulates Angiogenesis and Myogenesis During Post-Natal Skeletal Muscle Regeneration." J Cell Mol Med 13, no. 8B (2009): 2424-35.

26. Piccioni, A., E. Gaetani, V. Neri, I. Gatto, M. Palladino, M. Silver, R. C. Smith, I. Giarretta, E. Pola, L. Hlatky, and R. Pola. "Sonic Hedgehog Therapy in a Mouse Model of Age-Associated Impairment of Skeletal Muscle Regeneration." J Gerontol A Biol Sci Med Sci 69, no. 3 (2014): 245-52.

27. Palladino, M., I. Gatto, V. Neri, S. Straino, M. Silver, A. Tritarelli, A. Piccioni, R. C. Smith, E. Gaetani, D. W. Losordo, F. Crea, M. Capogrossi, and R. Pola. "Pleiotropic Beneficial Effects of Sonic 
Hedgehog Gene Therapy in an Experimental Model of Peripheral Limb Ischemia." Mol Ther 19, no. 4 (2011): 658-66.

28. Palladino, M., I. Gatto, V. Neri, E. Stigliano, R. C. Smith, E. Pola, S. Straino, E. Gaetani, M. Capogrossi, G. Leone, L. Hlatky, and R. Pola. "Combined Therapy with Sonic Hedgehog Gene Transfer and Bone Marrow-Derived Endothelial Progenitor Cells Enhances Angiogenesis and Myogenesis in the Ischemic Skeletal Muscle." J Vasc Res 49, no. 5 (2012): 425-31.

29. Qin, Y., Y. H. He, N. Hou, G. S. Zhang, Y. Cai, G. P. Zhang, Q. Xiao, L. S. He, S. J. Li, Q. Yi, and J. D. Luo. "Sonic Hedgehog Improves Ischemia-Induced Neovascularization by Enhancing Endothelial Progenitor Cell Function in Type 1 Diabetes." Mol Cell Endocrinol 423 (2016): 30-9.

30. Lavine, K. J., A. Kovacs, and D. M. Ornitz. "Hedgehog Signaling Is Critical for Maintenance of the Adult Coronary Vasculature in Mice." J Clin Invest 118, no. 7 (2008): 2404-14.

31. Paulis, L., J. Fauconnier, O. Cazorla, J. Thireau, R. Soleti, B. Vidal, A. Ouille, M. Bartholome, P. Bideaux, F. Roubille, J. Y. Le Guennec, R. Andriantsitohaina, M. C. Martinez, and A. Lacampagne. "Activation of Sonic Hedgehog Signaling in Ventricular Cardiomyocytes Exerts Cardioprotection against Ischemia Reperfusion Injuries." Sci Rep 5 (2015): 7983.

32. Xiao, Q., N. Hou, Y. P. Wang, L. S. He, Y. H. He, G. P. Zhang, Q. Yi, S. M. Liu, M. S. Chen, and J. D. Luo. "Impaired Sonic Hedgehog Pathway Contributes to Cardiac Dysfunction in Type 1 Diabetic Mice with Myocardial Infarction." Cardiovasc Res 95, no. 4 (2012): 507-16.

33. Kusano, K. F., R. Pola, T. Murayama, C. Curry, A. Kawamoto, A. Iwakura, S. Shintani, M. Ii, J. Asai, T. Tkebuchava, T. Thorne, H. Takenaka, R. Aikawa, D. Goukassian, P. von Samson, H. Hamada, Y. S. Yoon, M. Silver, E. Eaton, H. Ma, L. Heyd, M. Kearney, W. Munger, J. A. Porter, R. Kishore, and D. W. Losordo. "Sonic Hedgehog Myocardial Gene Therapy: Tissue Repair through Transient Reconstitution of Embryonic Signaling." Nat Med 11, no. 11 (2005): 1197-204.

34. Bijlsma, M. F., P. J. Leenders, B. J. Janssen, M. P. Peppelenbosch, H. Ten Cate, and C. A. Spek. "Endogenous Hedgehog Expression Contributes to Myocardial Ischemia-Reperfusion-Induced Injury." Exp Biol Med (Maywood) 233, no. 8 (2008): 989-96.

35. Ahmed, R. P., K. H. Haider, J. Shujia, M. R. Afzal, and M. Ashraf. "Sonic Hedgehog Gene Delivery to the Rodent Heart Promotes Angiogenesis Via Inos/Netrin-1/Pkc Pathway." PLoS One 5, no. 1 (2010): e8576.

36. Guo, W., X. Yi, F. Ren, L. Liu, S. Wu, and J. Yang. "Activation of Shh Signaling Pathway Promotes Vasculogenesis in Post-Myocardial Ischemic-Reperfusion Injury." Int J Clin Exp Pathol 8, no. 10 (2015): 12464-72.

37. Carbe, C. J., L. Cheng, S. Addya, J. I. Gold, E. Gao, W. J. Koch, and N. A. Riobo. "Gi Proteins Mediate Activation of the Canonical Hedgehog Pathway in the Myocardium." Am J Physiol Heart Circ Physiol 307, no. 1 (2014): H66-72.

38. Sims, J. R., S. W. Lee, K. Topalkara, J. Qiu, J. Xu, Z. Zhou, and M. A. Moskowitz. "Sonic Hedgehog Regulates Ischemia/Hypoxia-Induced Neural Progenitor Proliferation." Stroke 40, no. 11 (2009): 3618-26.

39. He, Q. W., Y. P. Xia, S. C. Chen, Y. Wang, M. Huang, Y. Huang, J. Y. Li, Y. N. Li, Y. Gao, L. Mao, Y. W. Mei, and B. Hu. "Astrocyte-Derived Sonic Hedgehog Contributes to Angiogenesis in Brain Microvascular Endothelial Cells Via Rhoa/Rock Pathway after Oxygen-Glucose Deprivation." Mol Neurobiol 47, no. 3 (2013): 976-87.

40. Dai, R. L., S. Y. Zhu, Y. P. Xia, L. Mao, Y. W. Mei, Y. F. Yao, Y. M. Xue, and B. Hu. "Sonic Hedgehog Protects Cortical Neurons against Oxidative Stress." Neurochem Res 36, no. 1 (2011): 67-75.

41. He, W., L. Cui, C. Zhang, X. Zhang, J. He, Y. Xie, and Y. Chen. "Sonic Hedgehog Promotes Neurite Outgrowth of Cortical Neurons under Oxidative Stress: Involving of Mitochondria and Energy Metabolism." Exp Cell Res 350, no. 1 (2017): 83-90.

42. Ji, H., J. Miao, X. Zhang, Y. Du, H. Liu, S. Li, and L. Li. "Inhibition of Sonic Hedgehog Signaling Aggravates Brain Damage Associated with the Down-Regulation of Gli1, Ptch1 and Sod1 Expression in Acute Ischemic Stroke." Neurosci Lett 506, no. 1 (2012): 1-6.

43. Ding, X., Y. Li, Z. Liu, J. Zhang, Y. Cui, X. Chen, and M. Chopp. "The Sonic Hedgehog Pathway Mediates Brain Plasticity and Subsequent Functional Recovery after Bone Marrow Stromal Cell Treatment of Stroke in Mice." J Cereb Blood Flow Metab 33, no. 7 (2013): 1015-24. 
44. Zhang, L., M. Chopp, D. H. Meier, S. Winter, L. Wang, A. Szalad, M. Lu, M. Wei, Y. Cui, and Z. G. Zhang. "Sonic Hedgehog Signaling Pathway Mediates Cerebrolysin-Improved Neurological Function after Stroke." Stroke 44, no. 7 (2013): 1965-72.

45. Jin, Y., A. Barnett, Y. Zhang, X. Yu, and Y. Luo. "Poststroke Sonic Hedgehog Agonist Treatment Improves Functional Recovery by Enhancing Neurogenesis and Angiogenesis." Stroke 48, no. 6 (2017): 1636-45.

46. Huang, S. S., H. Cheng, C. M. Tang, M. W. Nien, Y. S. Huang, I. H. Lee, J. H. Yin, T. B. Kuo, C. C. Yang, S. K. Tsai, and D. I. Yang. "Anti-Oxidative, Anti-Apoptotic, and Pro-Angiogenic Effects Mediate Functional Improvement by Sonic Hedgehog against Focal Cerebral Ischemia in Rats." Exp Neurol 247 (2013): 680-8. 